\title{
II.
}

Aus der medicinischen Klinik des Herrn Professor A. v. Strü̈MPeli in Erlangen.

\section{Ein Fall von Brown-Séquard'scher Halbseitenlähmung nach Stichverletzung des Rückenmarks.}

\author{
Von \\ Dr. med. Wilhelm Fürnrohr, \\ Volontär-Assistent.
}

(Mit 3 Figuren im Text.)

\begin{abstract}
Seitdem Brown-Séquard die ersten Untersuchungen über halbseitige Durchschneidung des Rückenmarks veröffentlichte, sind seine Experimente des Oeftern wiederholt worden, und zahlreiche Beobachtungen am Krankenbett haben die Ergebnisse des Thierexperiments ergänzt und erweitert. Wenn ich nun den vielen Fällen, in denen bereits, sei es nun durch Tumorbildung, sei es durch zufällige Verletzung des Rückenmarks, der Brown-Séquard'sche Symptomencomplex beobachtet wurde, einen weiteren der letzteren Art hinzufüge, so thue ich es, weil der Fall an sich verschiedene interessante Einzelheiten darbietet und mir gleichzeitig Gelegenheit giebt, auf verschiedene noch nicht hinreichend bekannte oder bisher nicht genügend gewürdigte Momente hinzuweisen.

Ich lasse zunächst einen kurzen Auszug aus der Krankengeschichte folgen.
\end{abstract}

\section{Anamnese.}

Mit 11 Jahren hatte Pat. eine nicht genauer bekannte Krankheit, die mit Schwellung der Beine einherging; mit 19 Jahren Lungenentzündung, mehrere Male Halsentzündung; sonst war P. immer gesund.

Potatorium sowie geschlechtliche Infection negirt.

Familienanamnese ergiebt nichts von Bedentung.

In der Nacht vom 5. zum 6. October 1901 war P. unfreiwillig an einer Rauferei betheiligt, in deren Verlauf er von hinten her einen mit grosser Gewalt geführten Stich in den Rücken erhielt. Fast gleichzeitig bekam er auch einen Stoss gegen die rechte Hüfte, durch den er zu Boden geworfen wurde. Zunächst war er nun etwa 10 Minuten bewusstlos nnd kam erst wieder zu sich, nachdem ihm sein in der Nähe befindlicher Sohn 
bereits das fest im Rücken steckende Messer herausgezogen hatte. Sofort merkte er, dass er das rechte Bein absolut nicht bewegen konnte (,es lag wie todt am Boden"), ausserdem war die ganze rechte Seite bis zur Brustwarze "pelzig" ; P. hatte kein Gefühl.

$P$. wurde nun ins Fürther Spital gebracht und unter geeigneter Behandlung heilte die Wunde am Rücken innerhalb weniger Tage.

Das rechte Bein jedoch blieb auch in den nächsten Wochen - P. war inzwischen in seine Privatwohnung verbracht worden - vollständig. gelähmt und gefühllos. Ebenso bestand auch von Anfang an ein schmerzhaftes Drücken auf der rechten Seite des Körpers - etwa wio ein festgeschnürtes Band - , das sich von der Gegend der Lendenwirbelsäule nach vorn zur Nabelgegend erstreckte. Der stärkste Schmerz war in und unterhalb der Nabelgegend und strahlte auch noch in die obere Hälfte des rechten Oberschenkels aus.

In den nächsten 8 Wochen soll sich nun im Befund gar nichts geändert haben.

In der 9. Woche etwa konnte $P$. mit dem rechten Bein ganz kleine Bewegungen machen. Um diese Zeit kehrte angeblich auch das Gefühl im. rechten Bein wieder zurïck. Gelegentlich eines Verbandes mit heissem Wasser machte $P$. auch die ihm sehr auffällige Wahrnehmung, dass heisses Wasser am rechten Bein ziemlich stark brannte, am linken dagegen absolut kein deutliches Wärmegefühl hervorrief. Ausserdem hatte er auch immer das Gefühl, dass das r. Bein warm, das linke kalt sei. "Er musste sich oft mit den Händen überzengen, dass auch das linke warm sei."

Blasenbeschwerden hatte P. nur einmal ganz vorïbergehend, dagegen bestand meist hartnäckige Verstopfung.

Erectionen sollen theils unvollständig, in der späteren Zeit gar nicht mehr aufgetreten sein.

Nachdem sich die Beweglichkeit des rechten Beines in der nächsten Zeit unter Behandlung mit dem faradischen Strom noch etwas gebessert, kam P. am 7. Februar auf die hiesige chirurgische Klinik nnd wurde von dort am 14. Februar auf die medicinische verlegt.

Status praesens. P.ist ein grosser, kräftig gebauter Mann in gutem Ernährungszustand. Musculatur und Fettpolster im Allgemeinen gut entwickelt.

Von Seiten der Gehirnnerven nichts Pathologisches.

Die Pupillen reagiren prompt auf Lichteinfall. Befund.

Die Untersuchung der inneren Organe ergiebt vollständig normalen

Urin ohne E. und $Z$. Beim Uriniren keine Beschwerden.

Am Rücken in der Höhe des 2. Brustwirbels $6 \mathrm{~cm}$ nach rechts von der Wirbelsäule eine ca. $2 \mathrm{~cm}$ lange, quer verlaufende glatte Hautnarbe, die anf der Unterlage gut verschieblich ist. normal.

Motilität. Bewegungen des Kopfes und beider Arme vollständig.

Rechtes Bein stark paretisch, so dass das Gehen ohne Stütze z. Z. gavz unmöglich ist; nur mit Hülfe von 2 Krücken kann sich P. ziemlich müllsam und langsam vorwärts bewegen. Das linke Bein erscheint dabei intact, das rechte jedoch schleift etwas nach und wird nur wie vorsichtig tastend, meist mit den Zehen zuerst, anf den Boden gesetzt. 
Im rechten Bein leichte Ataxie.

Ober- und Unterschenkel rechts etwas atrophisch.

Reflexe: An den Armen vorhanden, sicher nicht gesteigert, eher etwas schwach.

Bauchdeckenreflex links deutlich vorhanden, rechts nicht auszulösen.

Cremasterreflex links lebhaft, rechts nicht vorhanden.

Fusssoblenreflexe beiderseits vorhanden. Kein dentlicher Unterschied zwischen links und rechts.

Patellarreflex links ziemlich lebhaft, rechts stark gesteigert.

Achillesrefiex links lebhaft, rechts stark gesteigert. Starker Patellarund Fussclonus rechts.

Rechts Babinsky'scher Zehenreflex (Dorsalfiexion).

Tibialisphänomen rechts, links nicht vorhanden.

Zehenphänounen rechts, links nicht vorhanden.

Sensibilität: Tastempfindung am ganzen Körper gut, nur an einer bandförmigen Zone auf der rechten Körperhälfte schlecht.

Schmerzempfindung auf der linken Körperhälffte etwa vom Nabel nach abwärts schlecht, auf der rechten Seite gut, nur an der obenerwähnten bandförmigen Zone ebenfalls schlecht.

Temperaturempfindung auf der linken Körperhälfte in derselben Ausdehnung schlecht, rechts gut, in der bandförmigen Zone schlecht.

Drucksinn links intact, rechts von der oberen Grenze der bandförmigen Zone nach abwärts erheblich gestört.

Lagegefühl links intact, rechts in Hüft-, Knie-, Fussgelenk und in sämmtlichen Zehengelenken sehr schlecht.

Lagegefühl auch im Penis schlecht.

Das subjective Befinden des $P$. ist immer gut, nur klagt er sehr oft über theils drückende, theils brennende Schmerzen in der rechten Körperhälfte; auch das Gefühl, dass das r. Bein warm, das linke kalt sei, besteht noeh.

Wenn ich nun im Folgenden auf die vorstehende Krankengeschichte etwas näher eingehe, so ist zunächst der Anamnese nicht viel hinzuzufügen. Dass das spätere Leiden nur auf eine durch den Messerstich herbeigeführte Verletzung des Rückenmarks zurückgeführt werden kann, ist wohl ohne Weiteres klar.

Die interessante Angabe des Pat., dass in der ersten Zeit nach der Verletzung auch das Gefühl im rechten Bein vollständig erloschen gewesen sei, lässt sich leider, da eine ärztliche Beobachtung hierüber nicht vorliegt, nicht weiter verwerthen.

Auf die angegebenen subjectiven Empfindungen werde ich weiter unten noch zurückkommen.

Dem oben nur in den Hauptsachen skizzirten Befund, der sich während eines etwa dreiwöchentlichen Aufenthaltes des Pat. in hiesiger Klinik nur unwesentlich verändert hat, möchte ich zur weiteren Ausführung noch Verschiedenes hinzufügen.

Was zunächst die Verletzung selbst anlangt, so finden wir als Deutsche zeitschr. f. Nervenheilkunde. XXII. Bd. 
deren Residuen eine ganz kleine, unscheinbare Hautnarbe, $6 \mathrm{~cm}$ nach rechts von der Mittellinie, also eigentlich, wenn man bedenkt, dass der Stich von einem ziemlich kleinen Messer herrührte, relativ weit von dem geschädigten Rückenmark entfernt. Wir können uns diese Verhältnisse nicht anders erklären, als dass Pat. in dem Momente, wo er den Stich erhielt, eine kräftige Drehung des Arms - vielleicht schon als Abwehrbewegung - nach hinten vollführte und dadurch die Stelle der jetzigen Narbe der Medianlinie näher brachte.

Die Folgen der Verletzung des Rückenmarks machten sich nun nach zwei Richtungen hin geltend, wir konnten einerseits starke Störungen der Motilität, andererseits nicht mindere der Sensibilität beobachten. Entsprechend dem Sitze der Verletzung im oberen Brustmark waren Kopf und Arme natürlich frei beweglich und im ungestörten Besitze ihres normalen Gefühls, wir hatten es - zunächst motorisch - nur mit einer Lähmung des rechten Beins zu thun. Eine Betheiligung der Thorax- und Abdominalmusculatur oder des Zwerchfells an der Lähmung konnten wir nicht feststellen. $\mathrm{Ob}$ das rechte Bein nun, wie Pat. angab, früher vollständig gelähmt und absolut unbeweglich war, weiss ich nicht; bei uns jedenfalls konnte Pat. schon wieder eine ganze Reihe von Bewegungen, wenn auch z. Th. mit recht geringer Kraft, ausführen. Dabei zeigte sich nun, was auch bei anderen Krankheiten schon verschiedentlich beobachtet wurde, dass die Lähmung in viel höherem Grade die Beuge- als die Streckmusculatur befallen hatte. Während Pat. z. B. das Knie - hier trat dies am deutlichsten hervor - ausgezeichnet strecken konnte, war eine Beugung noch fast ganz unmöglich. Liess man den Pat. auf dem linken Bein - dem gesunden - stehen und hob nun den rechten Unterschenkel nach hinten in die Höhe, so fiel derselbe, trotz der grössten Anstrengungen des sehr willigen Pat., sofort wieder fast ganz herunter.

Auf diese eigenthümlichen Verhältnisse ist es wohl auch zurückzuführen, dass sich Pat. im Bett, ohne die Hände zu Hülfe zu nehmen, nicht aufsetzen konnte. Beim Versuch dazu spannte sich zwar die Bauchmusculatur gut an, aber Pat. konnte sich infolge der Schwäche der Beugemusculatur des Beins - wenn ich so sagen darf - auf der Unterlage nicht genügend „einhaken“. Statt des Rumpfes gingen die Beine in die Höhe. Drückte man aber die Beine an die. Unterlage fest an - ersetzte man dadurch sozusagen die Aufgabe der Beuger - so ging das Aufrichten glatt von Statten.

Wie nicht anders zu erwarten, war es natürlich auch bereits zu einer deutlichen Atrophie des rechten Beines gekommen. Die genauen Maasse geben hierüber den besten Aufschluss. 


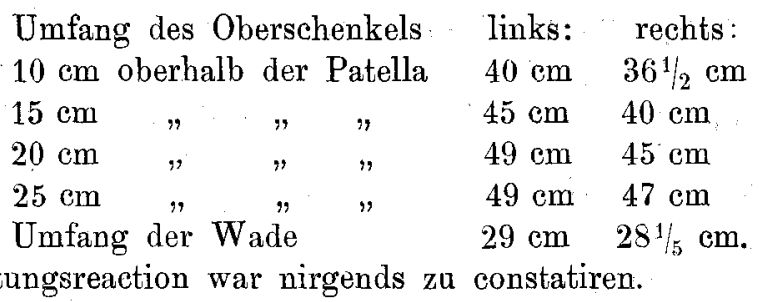

Bei Betrachtung der Reflexe konnten wir feststellen, wie auch trühere Beobachter, dass Bauchdeckenreflex und Cremasterreflex auf der gesunden Seite vorhanden waren, auf der Seite der Verletzung aber fehlten. Es kann uns dies nicht wundern, wenn wir, wie dies neuerdings geschieht, annehmen, dass die beiden zugehörigen Reflexbögen sehr hoch oben im Rückenmark bez. Gehirn liegen.

Der Fusssohlenreflex war wohl beiderseits vorhanden. Die Sehnenreflexe, die schon links ziemlich lebhaft waren, zeigten sich rechts ausserordentlich gesteigert; beim geringsten Beklopfen der Patellaroder Achillessehne, ja schon beim Beklopfen der Tibiakante, trat deutliche Reaction ein. Patellar- und Fussclonus waren natürlich rechts ebenfalls sehr leicht auszulösen. Letzterer war dadurch, dass er schon bei sehr geringen Reizen eintrat, für den Pat., dem das häufige "Zittern" sehr unangenehm war, ausserordentlich lästig.

Auch der Babinsky'sche Zehenreflex war in unserem Falle gut ausgeprägt. Beim Bestreichen der Fusssohle mit dem Stiele des Percussionshammers trat links Plantarflexion, rechts deutliche, langsame Dorsalflexion der grossen Zehe ein.

Gleich hier möchte ich auch noch auf zwei Symptome etwas näher eingehen, die von Strümpell ${ }^{1}$ ) in die Neurologie eingeführt wurden, und die wir in unserem Falle sehr schön beobachten konnten: das Tibialis- und das Zehenphänomen. Das Tibialisphänomen, dessen Auftreten Strümpell auf eine Erkrankung der Pyramidenbahn zurückführt, besteht darin, dass beim Anziehen des paretischen Beines an den Rumpf, wodurch eine Beugung im Hüft- und Kniegelenk herbeigeführt wird, ,sofort eine sicht- und fühlbare Anspannung der Sehne des Tibialis anticus am Fussrücken eintritt", während beim Gesunden "der Fuss in schlaffer Plantarflexionsstellung bleibt". Diese Erscheinung, die Strümpell bei multipler Sklerose, Rückenmarkstumoren, myelitischen Lähmungen und anderen Krankheiten, die zu spastischer Parese der Beine führten, nicht aber bei functionellen Lähmungen beobachtete, war auch bei unserem Pat. sehr deutlich vorhanden. Be-

1) Diese Zeitschrift. Bd. XX, S. 436. 
sonders stark trat die Anspannung der Tibialissehne und damit auch die Hebung des inneren Fussrandes hervor, wenn man, wie dies Strümpell angiebt, der Beugung des Beins durch die auf dem Oberschenkel ruhende Hand einen gewissen Widerstand entgegensetzte.

Das Zehenphänomen, das darin besteht, dass beim Erheben des gestreckten paretischen Beins eine starke Dorsalflexion der grossen Zehe auftritt, war auf diese Weise nicht zu erzielen, trat aber sofort deutlich auf, wenn man das gebeugte Bein ausstrecken liess und dabei einen geringen Gegendruck ausübte.

Während nun die im Vorhergehenden geschilderten Störungen der Motilität, die Parese des Beins, in Sonderheit der Beugemusculatur, die Steigerung der Sehnenreflexe bei anfgehobenen Hautreflexen, end-

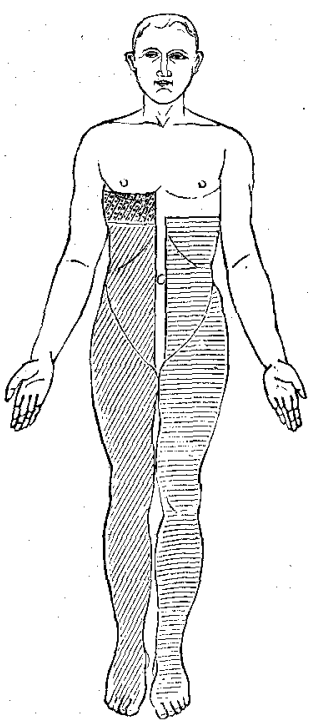

Fig. 1.

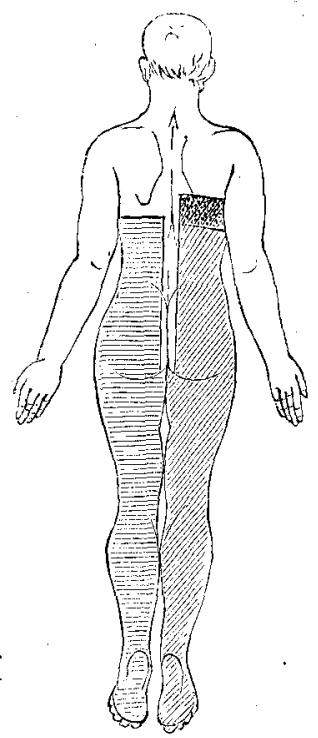

Fig. 2.

lich das Tibialis- und Fussphänomen lediglich auf der rechten Seite des Körpers zu finden waren, bei völliger Intactheit der linken, bieten sich uns nunmehr bei Betrachtung der Sensibilitätsstörungen viel complicirtere Verhältnisse dar.

Wie schon früher andere Beobachter konnten auch wir zunächst eine anästhetische - - in unserem Falle besser hypästhetische Zone etwas unterhalb der verletzten Stelle des Rückenmarks feststellen, an der das Gefühl für alle Qualitäten zwar nicht vollständig aufgehoben, aber bedeutend herabgesetzt war. Diese lag natürlich auf der 
rechten Körperhälfte und zog als etwa $10-11 \mathrm{~cm}$ breites Band, dessen obere Grenze vorne etwa $3 \mathrm{~cm}$ unterhalb der Brustwarze, hinten etwa in Höhe des 5. B.-W. lag, von vorne nach hinten. Die Medianlinie wurde von dieser Zone nirgends erreicht, vielmehr war etwa $2-3 \mathrm{~cm}$ nach rechts von derselben noch ganz gutes Gefühl nachzuweisen.

Am schlechtesten waren hier Tastempfindung und Drucksinn, etwas besser, aber ebenfalls noch deutlich gestört Schmerz- und Temperatursinn; Lagegefühl konnte naturgemäss an dieser Körperstelle nicht in Betracht kommen. Diese Zone, an der im Gegensatz zu den späteren Befunden das Gefühl für alle Qualitäten herabgesetzt ist, entspricht wohl den durch den Stich verletzten Wurzeln; dass dieselbe etwas tiefer liegt, als man vielleicht erwarten konnte, entspricht einer bekannten Erfahrungsthatsache.

Die weiterhin noch beobachteten Sensibilitätsstörungen vertheilen sich nun anf den Rumpf und die beiden unteren Extremitäten, und zwar so, dass Schmerz- und Temperatursinn auf der linken, Drucksinn und Lagegefühl auf der rechten Seite herabgesetzt erscheinen. Das Gefühl für einfache Berührung, das nach den Angaben des Pat. wohl in der ersten Zeit nach der Verletzung ebenfalls beträchtlich gestört gewesen sein muss, zeigte sich bei uns auf beiden Seiten gleich gut erhalten; die einzige Ausnahme machte die schon erwähnte bandförmige Zone; ausserdem war nur noch ein nicht sehr grosser Bezirk unterhalb derselben für Berührung vielleicht etwas weniger empfindlich.

Die Herabsetzung des Temperatur- und Schmerzgefühls auf der linken Körperhälfte fand ihre obere Grenze vorne etwa $9 \mathrm{~cm}$ unterhalb der Mamilla, hinten etwa am 9. B.-W., dieselbe lag also wesentlich tiefer als die Verletzung des Rückenmarks. Die Begrenzung nach rechts lag etwas vor der Mittellinie. An dieser war bereits wieder ganz normales Gefühl. Nach unten zu war die Empfindung im ganzen Bein herabgesetzt; auch Penis und Scrotum waren in zwei verschieden empfindende Hälften getheilt.

Der Drucksinn war, wie bereits erwähnt, schon oben in der bandförmigen Zone der verletzten Wurzelgebiete deutlich herabgesetzt und diese Herabsetzung setzte sich continuirlich nach unten auf die rechte Thoraxhälfte und das rechte Bein fort; auch Penis und Scrotum waren wiederum in zwei Hälften getheilt. An der Mittellinie war das Gefühl für Druck bereits wieder normal.

Das Gefühl für passive Bewegungen zeigte sich im rechten Bein beträchtlich herabgesetzt, und zwar so, dass die ausgeführten Bewegungen am schlechtesten in den distal gelegenen Gelenken - den Zehengelenken, dem Fussgelenk - erkannt wurden; entschieden besser empfanden noch Knie- und Hüftgelenk. Auch hier handelte es sich 
nicht um eine totale Aufhebung des Lagegefühls, sondern nur um eine beträehtliche Verminderung: ganz grosse Bewegungen wurden in den verschiedenen Gelenken meist noch ganz richtig erkannt. Dieser Herabsetzung des Lagegefühls ist es wohl auch zuzuschreiben, dass sich bei Bewegungen des rechten Beins, besonders des Unterschenkels eine freilich nicht sehr hochgradige Ataxie bemerkbar machte.

Eine ganz ähnliche Thatsache konnte ich auch am Penis feststellen. Während Gesunde mit dem Penis ausgeführte passive Bewegungen ausnahmslos richtig localisiren, machte unser Kranke fast mmer falsche Angaben.

Fassen wir den ganzen Befund unserer Sensibilitätsprüfung zusammen, so sehen wir, dass wir es auch in unserem Falle mit einer sog. gekreuzten Lähmung zu thun haben; auf der Seite der motorischen Lähmung sind Drucksinn und Lagegefühl herabgesetzt, auf der nicht gelähmiten Schmerz- und 'Temperatursinn. Auch unser Fall spricht also dafür, dass sich die Fasern für Schmerz und Temperatur sofort nach ihrem Eintritt ins Rückenmark, die, vielleicht identischen, für Drucksinn und Lagegefühl erst höher oben kreuzen. Die Streitfrage, ob dem Tastsinn nur eine oder mehrere Leitungsbahnen im Rückenmark zur Verfügung stehen, kann ich nicht entscheiden; jedenfalls spricht die Thatsache, dass bei uns, relativ kurze Zeit nach der Verletzung, das Gefühl für einfache Berührung fast am ganzen Körper intact war, zum Mindesten nicht gegen die letztere Annahme.

Gelegentlich der Beschreibung der bandförmigen Zone wurde bereits erwähnt, dass dieselbe nicht ganz bis zur Nittellinie reichte; genau dasselbe zeigte sich nun auch (cf. oben!) bei Prüfung der übrigen Qualitäten; dies Verhältniss illustrirt sehr schön die bekannte Thatsache, dass das sensible Innervationsgebiet einer Körperhälfte nicht genau in der Mittellinie aufhört, sondern sich noch einige Centimeter auf die andere Körperhälfte fortsetzt; die Mittellinie und deren nächste Umgebung empfängt somit ihre Nerven aus beiden Rückenmarkshälften.

Eine deutliche Hyperästhesie, die in anderen Fällen verschiedentlich beobachtet wurde, konnten wir nirgends mit Sicherheit feststellen. Zuweilen machte es freilich den Eindruck, als ob auf der rechten Körperhälfte, nach abwärts von der bandförmigen Zone, Nadelstiche etwas schmerzhafter, "Warm" und "Kalt" etwas intensiver empfunden würden, als oben an den Armen oder im Gesicht, also an sicher normaler Hautstelle.

Soweit der objective Befund.

Mit einigen Worten möchte ich nun noch auf die mehrfach erwähnten subjectiven Empfindungen des Pat. zurückkommen. Schon 
kurz nach der Verletzung bestanden angeblich drückende, zusammenschnürende Schmerzen auf der rechten Thoraxhälfte, die sich auch poch auf den rechten Oberschenkel fortsetzten.

Dieselben bestanden anfänglich vorne und hinten gleich stark, besserten sich aber allmählich, so dass wir bei uns durch genaues Fragen nur mehr Folgendes eruiren konnten (s. Abbildung 3): Von der rechten Brustwarze bis etwa zum Rippenbogen und von der vorderen Axillarlinie bis fast zur Mittellinie (also nur vorne) besteht eine Zone, an der Pat. immer Schmerzen hat. Dieselben werden als zusammenschnürende bezeichnet. Nach unten zu kommt nun ein schmaler Streifen mit ganz normalem Gefühl, dann wiederum ein Gebiet mit "brennenden" Schmerzen, das sich nach unten bis zur Mitte des Oberschenkels erstreckt. Diese "brennenden" Schmerzen strahlen auch nach oben aus und ziehen zuweilen, die Mittellinie überschreitend, ins linke Hypogastrium. Aller Wahrscheinlichkeit nach handelt es sich hierbei um. Schmerzen, die extraspinal in der Verletzung der Nerven-

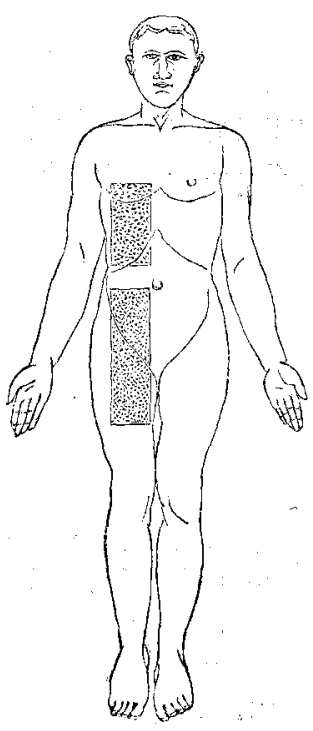

Fig. 3. wurzeln ihre Ursache haben. Vielleicht spielen auch, gerade für die untere Schmerzzone möchte ich daran denken, Parästhesien hierbei eine gewisse Rolle. Ins letztere Gebiet gehören wohl auch die Angaben des Pat, ,er habe das Gefühl, als ob das rechte Bein warm, das linke kalt sei", "um so mehr, als sich dies in den letzten Tagen vor der Entlassung in das Gegentheil verkehrte. Einen wirklichen Temperaturunterschied zwischen links und rechts, wie er anderweitig schon beobachtet wurde, konnten wir nicht feststellen.

Für die Ueberlassung des interessanten Falles zur Publication möchte ich auch an dieser Stelle meinem verehrten Chef und Lehrer, Herrn Prof. Dr. v. Strümpell, meinen verbindlichsten Dank aussprechen. 